University of Wollongong

Research Online

Faculty of Engineering and Information

Faculty of Engineering and Information

Sciences - Papers: Part A

Sciences

$1-1-2013$

Strain estimation of CFRP-confined concrete columns using energy approach

Thong M. Pham

University of Wollongong, tmp@uow.edu.au

Muhammad N.S Hadi

University of Wollongong, mhadi@uow.edu.au

Follow this and additional works at: https://ro.uow.edu.au/eispapers

Part of the Engineering Commons, and the Science and Technology Studies Commons

Research Online is the open access institutional repository for the University of Wollongong. For further information contact the UOW Library: research-pubs@uow.edu.au 


\title{
Strain estimation of CFRP-confined concrete columns using energy approach
}

\begin{abstract}
A new model is presented for calculating the axial strain of carbon fiber-reinforced polymer (CFRP)confined concrete columns. An energy-balance approach is introduced to establish a relationship of the energy absorption between a confined concrete column and CFRP. The proposed model was verified using a large database collected from 167 CFRP-confined plain concrete specimens. This database contains 98 circular specimens with diameters ranging between 100 and $152 \mathrm{~mm}$, and 69 square specimens having a side length ranging between 100 and $152 \mathrm{~mm}$. The database covers unconfined concrete strengths from 20 to $50 \mathrm{MPa}$. The proposed model shows very good correlation with the experimental results. In addition, the proposed model also provides a comparative prediction of the strain of CFRP-confined concrete columns in two extreme cases, i.e., (1) insufficient confinement, and (2) heavy confinement, which are not usually well predicted by other models.
\end{abstract}

\section{Disciplines}

Engineering | Science and Technology Studies

\section{Publication Details}

Pham, T. M. \& Hadi, M. N.S. (2013). Strain estimation of CFRP-confined concrete columns using energy approach. Journal of Composites for Construction, 17 (6), 04013001-1-04013001-11. 


\title{
Strain Estimation of CFRP-Confined Concrete Columns Using Energy Approach
}

\author{
Thong M. Pham, S.M.ASCE ${ }^{1}$; and Muhammad N. S. Hadi, M.ASCE ${ }^{2}$
}

\begin{abstract}
A new model is presented for calculating the axial strain of carbon fiber-reinforced polymer (CFRP)-confined concrete columns. An energy-balance approach is introduced to establish a relationship of the energy absorption between a confined concrete column and CFRP. The proposed model was verified using a large database collected from 167 CFRP-confined plain concrete specimens. This database contains 98 circular specimens with diameters ranging between 100 and $152 \mathrm{~mm}$, and 69 square specimens having a side length ranging between 100 and $152 \mathrm{~mm}$. The database covers unconfined concrete strengths from 20 to $50 \mathrm{MPa}$. The proposed model shows very good correlation with the experimental results. In addition, the proposed model also provides a comparative prediction of the strain of CFRP-confined concrete columns in two extreme cases, i.e., (1) insufficient confinement, and (2) heavy confinement, which are not usually well predicted by other models. DOI: 10.1061/(ASCE)CC.1943-5614.0000397. () 2013 American Society of Civil Engineers.
\end{abstract}

Author keywords: Carbon-fiber-reinforced polymer; Energy methods; Energy dissipation; Stress-strain relations.

\section{Introduction}

The use of carbon-fiber-reinforced polymers (CFRPs) in structural engineering has increased in recent years. However, estimating the capacity of FRP-confined concrete members is not very well-correlated with their actual behavior; as such, more attention must be paid to models for FRP-confined concrete. A complete model includes formulas to calculate the ultimate strength and ultimate strain of confined concrete and stressstrain relationships. Most of these studies have focused on the strength and stress-strain relationships of confined concrete. Not many models deal with strain prediction. Bisby et al. (2005) carried out an overview, and they conclude that existing models show a poor correlation with experimental results of confined concrete strain. Bisby et al. (2005) revealed that the mean absolute error of strain estimations ranges from 35 to $250 \%$, whereas the error of strength estimation is less than $20 \%$. The literature of FRP-confined concrete is excellent for calculating the confined concrete strength but not for calculating the corresponding strain.

Richart et al. (1929) reported that the axial strain at the compressive strength of confined concrete can be linearly related to the maximum confining pressure. Early studies, based on this assumption, that proposed formulas for strain estimation include Karbhari and Gao (1997), Miyauchi et al. (1999), Toutanji (1999), and Ilki et al. (2008). Another commonly used approach

${ }^{1}$ Ph.D. Candidate, School of Civil, Mining, and Environmental Engineering, Univ. of Wollongong, Wollongong, NSW 2522, Australia; formerly, Lecturer, Faculty of Civil Engineering, HCMC Univ. of Technology, Ho Chi Minh City 70000, Vietnam. E-mail: mtp027@ uowmail.edu.au

${ }^{2}$ Associate Professor, School of Civil, Mining, and Environmental Engineering, Univ. of Wollongong, Wollongong, NSW 2522, Australia (corresponding author). E-mail: mhadi@uow.edu.au

Note. This manuscript was submitted on November 25, 2012; approved on May 14, 2013; published online on May 16, 2013. Discussion period open until February 9, 2014; separate discussions must be submitted for individual papers. This paper is part of the Journal of Composites for Construction, (C) ASCE, ISSN 1090-0268/04013001(11)/\$25.00. is based on volume strain and dilation behavior (Lam and Teng 2003a, b) or regression analysis of experiments (Shehata et al. 2002). All of the previously noted studies used the mechanism behavior of confined concrete to obtain strain estimations. In addition, Mander et al. (1988) proposed an energy-balanced method to calculate the strain of steel confined concrete. This method assumes that the additional strain energy of a confined concrete column is equal to the energy used to fracture the hoops. A study by Saadatmanesh et al. (1994) adopted this method to calculate the strain of FRP-confined concrete. The writers' paper develops relationships between the additional energy absorption of a confined concrete column and the energy absorbed by the confinement material. A new methodology is introduced to calculate the confined concrete strain in circular and square sections.

\section{Analytical Investigation}

\section{Strain Energy and Energy Absorption}

Strain energy is the energy stored in a structural elastic member as a result of the work performed on the member by an external load. It is defined as the energy absorbed by the structural member during the loading process. For an axially loaded column, the work done by the applied load is equal to the area under the load-displacement curve [Fig. 1(a) and Eq. (1)]. In a similar manner, the energy absorbed by the external FRP in an FRP-confined concrete column can be estimated

$$
U=W=\int_{0}^{l} P d l
$$

where $U=$ strain energy; $W=$ work done by the applied load; $P=$ applied load; $l=$ displacement; and $d l$ is an increment of the displacement.

The energy stored in the column core is transferred to compress concrete, deform the FRP, create cracks in concrete, and vertically compress the FRP. Some energy is also lost in unknown consumptions. Because of the limited understanding of the behavior inside 
FRP-confined concrete, it would be inappropriate to directly use the balanced-energy approach proposed by Mander et al. (1988) for steel confined concrete. In this paper, it is assumed that there is a possible linear relationship between the energy absorption of the column and the external FRP, which is discussed in a subsequent section.

To further investigate the energy transfer, early studies focused on FRP tubes made from high-strength and high-stiffness fibers. Unlike ductile metals, fibers and resins are brittle and they fail by fracture after an initial elastic deformation. The fracture strain of a typical carbon fiber is around 1.5-2.0\% such that they may absorb less energy than conventional metals. However, they actually perform much better when a comparison is made in terms of the specific energy absorption, which is the energy per unit mass (Lu and Yu 2003). The specific energy absorption of fiber is affected by fiber strength, elastic properties, the diameter-to-thickness ratio of FRP, fiber orientation, and sectional geometries (Wolff et al. 1994). These studies confirm that directly using the balanced-energy method (proposed for steel confined concrete) for FRP-confined concrete is inappropriate.

\section{Energy in Structural Members}

The widely accepted model [Fig. 1(b)] is recommended by ACI 440-2R.08 (ACI 2008) for the stress-strain relationship of FRP-confined concrete columns. It was adopted in the writers' paper to calculate the energy absorption of a FRP-confined concrete column

$$
W_{c c}=A_{c c} \int_{0}^{\varepsilon_{c c}} f_{c} d \varepsilon_{c}
$$

where $W_{c c}=$ strain energy of confined concrete; $A_{c c}=$ grosssectional area of confined concrete; $f_{c}=$ stress of confined concrete; $\varepsilon_{c}=$ strain of confined concrete; $\varepsilon_{c c}=$ strain at peak stress of confined concrete; and $d \varepsilon_{c}$ is an increment of the axial strain.

The stress-strain curve [Fig. 1(b)] has been slightly modified to obtain a simple integration. An expression [Eq. (3)] was extracted from Eq. (2) to calculate the energy absorption of the concrete core, in which the volumetric strain energy equals the area under the experimental stress-strain curves. When the strain of confined concrete is less than the peak strain of the corresponding unconfined concrete, the effect of FRP is negligible. Thus, the writers assumed that the additional energy in the column core equals the area under the experimental stress-strain curves starting from the value of the unconfined concrete strain [Fig. 1(b) and Eq. (3)]

$$
U_{c c}=\int_{\varepsilon_{c o}}^{\varepsilon_{c c}} f_{c} d \varepsilon_{c}=\frac{\left(\varepsilon_{c c}-\varepsilon_{c o}\right)\left(f_{c o}^{\prime}+f_{c c}^{\prime}\right)}{2}
$$

where $U_{c c}=$ volumetric strain energy of confined concrete; $f_{c c}^{\prime}=$ confined concrete strength; $f_{c o}^{\prime}=$ unconfined concrete strength; and $\varepsilon_{c o}=$ its corresponding strain.

Similarly, the energy absorbed by FRP can be calculated

$$
W_{f}=\rho_{f} A_{c c}\left(0.5 f_{f} \varepsilon_{f}\right)
$$

where $W_{f}=$ strain energy of FRP; $f_{f}$ and $\varepsilon_{f}=$ rupture strength and rupture strain, respectively, of FRP obtained from flat-coupon tests; and $\rho_{f}=$ volumetric ratio of FRP as shown in Eqs. (5) and (6).

The volumetric ratio $\left(\rho_{f}\right)$ of FRP of circular and square sections can be calculated.
For circular sections

$$
\rho_{f}=\frac{4 t}{d}
$$

and for square sections

$$
\rho_{f}=\frac{t[4 b-r(8-2 \pi)]}{b^{2}-r^{2}(4-\pi)}=\Psi t
$$

where $t=$ thickness of FRP; $d=$ diameter of the section; and $r=$ radius of the round corner of the section.

The rupture strain of FRP on the confined concrete is much less than that obtained from flat-coupon tests (Xiao and Wu 2000; Pessiki et al. 2001; Carey and Harries 2005). Therefore, the volumetric strain energy of FRP on a column can be estimated as follows:

$$
U_{f}=\rho_{f}\left(0.5 f_{f e} \varepsilon_{f e}\right)
$$

where $U_{f}=$ volumetric strain energy of FRP; and $f_{f e}$ and $\varepsilon_{f e}=$ actual rupture strength and rupture strain, respectively, of FRP on the columns.

The energy absorbed by the column was calculated using Eq. (3) and the energy absorbed by FRP was estimated using Eq. (7). Next, a regression analysis based on a database was used to obtain a linear relationship between them. Based on that linear relationship, a model to calculate the strain of confined concrete at peak stress was derived.

\section{Experimental Database}

\section{Test Database}

Several experimental tests have been conducted on FRP-confined concrete by researchers over the past few decades. The writers collated a test database of 329 FRP confined plain concrete specimens reported by Demers and Neale (1994), Watanabe et al. (1997), Matthys et al. (1999), Rochette and Labossière (2000), Xiao and Wu (2000), Suter and Pinzelli (2001), Parvin and Wang (2001), Pessiki et al. (2001), Shehata et al. (2002), De Lorenzis et al. (2002), Karabinis and Rousakis (2002), Lam and Teng (2003b), Chaallal et al. (2003), Ilki and Kumbasar (2003), Masia et al. (2004), Berthet et al. (2005), Lam et al. (2006), Saenz and Pantelides (2006), Jiang and Teng (2007), Valdmanis et al. (2007), Al-Salloum (2007), Rousakis et al. (2007), Wang and Wu (2008), Tao et al. (2008), Wu and Wei (2010), Rousakis and Karabinis (2012), and Hadi et al. (2013). The primary focus of this paper is on CFRP; as such, test results of materials other than CFRP were excluded from this database. Moreover, test results of circular sections not reporting the actual rupture strain $\left(\varepsilon_{f e}\right)$ of FRP were excluded.

A few studies concluded that square columns confined with FRP provide a little (Mirmiran et al. 1998) or no (Wu and Zhou 2010) strength improvement. Thus, this paper deals only with round-corner square specimens; as such, specimens with sharp corners were excluded from the database. Because the procedure of calculating the strain of FRP-confined concrete is based on the ascending type of specimen (Fig. 1), the test results of square specimens that have a descending type were excluded from the database. After excluding all of the previously noted test results, the database contained the test results of 167 FRP confined plain concrete specimens, as follows: (1) 98 circular specimens, and (2) 69 square specimens. The circular specimens included in the database have diameters d ranging from 100 to $152 \mathrm{~mm}$ and have 


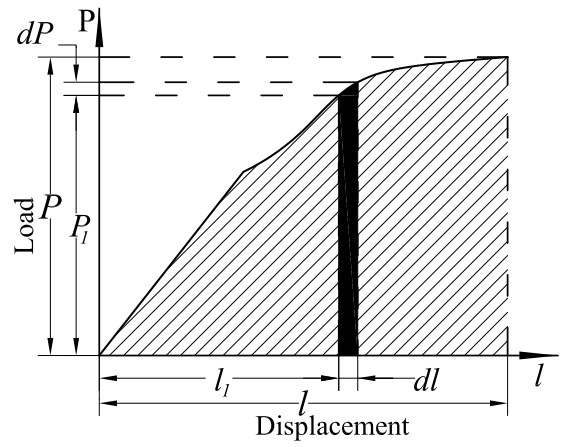

(a)

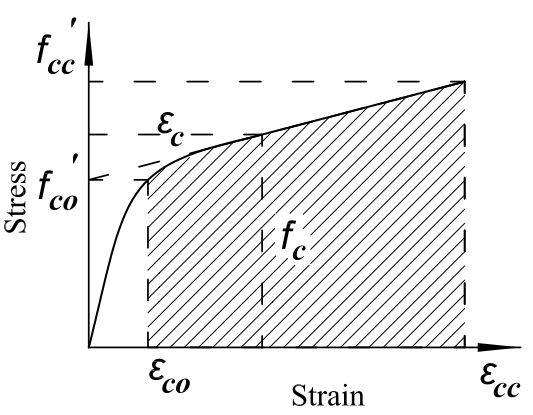

(b)

Fig. 1. (a) Load-displacement diagram; (b) typical stress-strain curve of FRP-confined concrete

unconfined concrete strengths $f_{c c}^{\prime}$ between 30 and $50 \mathrm{MPa}$. The square specimens have a side length ranging between 100 and $152 \mathrm{~mm}$, and unconfined concrete strength ranging between 20 and $50 \mathrm{MPa}$.

The confinement ratio was calculated by dividing the confining pressure $\left(f_{l}\right)$ by the unconfined concrete strength $\left(f_{c c}^{\prime}\right)$, which varied between 2 and $99 \%$ for circular specimens, and between 1 and $60 \%$ for square specimens. Tables 1 and 2 show the databases for circular and square specimens, respectively.

\section{Assumptions}

The actual rupture strain of CFRP is usually reported for circular sections but not for square sections. When the actual rupture strain of CFRP was not included in the test results, it was assumed to be 0.55 of the rupture strain from flat-coupon tests, as recommended by ACI 440.2R-08 (ACI 2008). In addition, when the axial strain at the peak stress of unconfined concrete $\left(\varepsilon_{c o}\right)$ was not specified, $\varepsilon_{c o}$ was assumed to be equal to 0.002 or values estimated by using the equation of Tasdemir et al. (1998). The performance of the proposed model was compared by using two different methods for estimating the values of $\varepsilon_{c o}$. In the first method, $\varepsilon_{c o}$ was calculated using the equation proposed by Tasdemir et al. (1998). In the second method, $\varepsilon_{c o}$ was assumed to be 0.002 . Results of using the first method proved to be better than the second method. Therefore, the equation proposed by Tasdemir et al. (1998) was used

$$
\varepsilon_{c o}=\left(-0.067 f_{c o}^{\prime 2}+29.9 f_{c o}^{\prime}+1,053\right) 10^{-6}
$$

\section{Proposed Strain Model}

A linear relationship was assumed between the energy absorbed by a column core and CFRP for both circular sections and square sections. The energy absorption was calculated using Eqs. (3) and (7), whereas a regression analysis was carried out to obtain an equation for the energy absorbed in the form shown in Eq. (9). Based on this regression analysis, a new formula is proposed to calculate the strain at the peak stress of CFRP-confined concrete

$$
U_{c c}-k U_{f}
$$

where $k$ is the proportion factor, which is a function of fiber stiffness and sectional geometries.

\section{Strain Estimation for Circular Sections}

The energy absorption of 98 circular specimens was estimated using Eqs. (3) and (7), and Fig. 2 presents the results. Next, a regression analysis was undertaken to attain

$$
U_{c c}=7.6 U_{f}
$$

Substituting Eqs. (3) and (7) into Eq. (10) results in Eq. (11)

$$
\varepsilon_{c c}=\varepsilon_{c o}+\frac{4 k t f_{f e} \varepsilon_{f e}}{d\left(f_{c o}^{\prime}+f_{c c}^{\prime}\right)}
$$

where the proportion factor $k=7.6$. Eq. (11) can be used to calculate the strain of CFRP-confined concrete columns in circular sections. Using this calculated strain, any model can be utilized to calculate the confined concrete strength. The Lam and Teng model (2003a) was adopted to express another form of Eq. (11)

$$
\varepsilon_{c c}=\varepsilon_{c o}+\frac{2 k t f_{f e} \varepsilon_{f e}}{d f_{c o}^{\prime}+3.3 f_{f e} t}
$$

\section{Strain Estimation for Square Sections}

For circular sections, the methodology proposed in this paper was used to establish a relationship between the energy absorption of the entire column section and FRP. The energy absorption of the FRP was calculated with respect to the perimeter of the section. This calculation did not provide a comparable correlation between the two energies in Eq. (9). Thus, the energy absorption of the column core at the effective area [Fig. 3(a)] was considered for the square specimens, which accounts for stress concentration at the corners. Details of the previously noted modifications are analyzed in the subsequent sections.

The energy absorption is sensitive to the geometry of the column (Wolff et al. 1994). Thus, equations simulating the relationship between the absorption energies of a column and CFRP distinguish square from circular specimens. In addition, the confining pressure of a square column confined with CFRP is not uniform. Karabinis et al. (2008) and Csuka and Kollár (2012) proved that the confining pressure primarily concentrates on round corners of the column, whereas this confining pressure is negligible at other zones [Fig. 3(b)]. Therefore, the energy absorption used to rupture CFRP is assumed to be only available at the round corners. In such a case, a corner energy ratio $k_{c}$, which is the ratio of the total length of four round corners [Fig. 3(c)] to the circumference of the section, is introduced to account for the reduction of energy absorbed by CFRP

$$
k_{c}=\frac{\pi r}{2 b-r(4-\pi)}
$$

where $b=$ side length of a square section; and $r=$ round radius at the corners of the section. 
Table 1. Database of Circular Specimens

\begin{tabular}{|c|c|c|c|c|c|c|c|c|c|c|c|}
\hline Number & Source of data & $\begin{array}{c}d \\
(\mathrm{~mm})\end{array}$ & $\begin{array}{c}h \\
(\mathrm{~mm})\end{array}$ & $\begin{array}{c}f_{c o}^{\prime} \\
(\mathrm{MPa})\end{array}$ & $\begin{array}{l}\varepsilon_{c o} \\
(\%)\end{array}$ & $\begin{array}{c}t \\
(\mathrm{~mm})\end{array}$ & $\begin{array}{c}f_{f} \\
(\mathrm{MPa})\end{array}$ & $\begin{array}{c}E_{f} \\
(\mathrm{GPa})\end{array}$ & $\begin{array}{l}\varepsilon_{c c} \\
(\%)\end{array}$ & $\begin{array}{l}\varepsilon_{f e} \\
(\%)\end{array}$ & $\begin{array}{c}f_{c c} \\
(\mathrm{MPa})\end{array}$ \\
\hline 1 & Watanable et al. (1997) & 100 & 200 & 30.2 & 0.23 & 0.17 & 2,716 & 225 & 1.51 & 0.94 & 46.6 \\
\hline 2 & Ibid & 100 & 200 & 30.2 & 0.23 & 0.50 & 2,873 & 225 & 3.11 & 0.82 & 87.2 \\
\hline 3 & Ibid & 100 & 200 & 30.2 & 0.23 & 0.14 & 1,579 & 629 & 0.57 & 0.23 & 41.7 \\
\hline 4 & Ibid & 100 & 200 & 30.2 & 0.23 & 0.28 & 1,824 & 630 & 0.88 & 0.22 & 56.0 \\
\hline 5 & Ibid & 100 & 200 & 30.2 & 0.23 & 0.42 & 1,285 & 577 & 1.30 & 0.22 & 63.3 \\
\hline 6 & Matthys et al. (1999) & 150 & 300 & 34.9 & 0.21 & 0.12 & 2,600 & 200 & 0.85 & 1.15 & 44.3 \\
\hline 7 & Ibid & 150 & 300 & 34.9 & 0.21 & 0.12 & 2,600 & 200 & 0.72 & 1.08 & 42.2 \\
\hline 8 & Ibid & 150 & 300 & 34.9 & 0.21 & 0.24 & 1,100 & 420 & 0.40 & 0.19 & 41.3 \\
\hline 9 & Ibid & 150 & 300 & 34.9 & 0.21 & 0.24 & 1,100 & 420 & 0.36 & 0.18 & 40.7 \\
\hline 10 & Rochette and Labossière (2000) & 100 & 200 & 42 & - & 0.60 & 1,265 & 83 & 1.65 & 0.89 & 73.5 \\
\hline 11 & Ibid & 100 & 200 & 42 & - & 0.60 & 1,265 & 83 & 1.57 & 0.95 & 73.5 \\
\hline 12 & Ibid & 100 & 200 & 42 & - & 0.60 & 1,265 & 83 & 1.35 & 0.80 & 67.6 \\
\hline 13 & Xiao and Wu (2000) & 152 & 305 & 33.7 & - & 0.38 & 1,577 & 105 & 1.20 & 0.84 & 47.9 \\
\hline 14 & Ibid & 152 & 305 & 33.7 & - & 0.38 & 1,577 & 105 & 1.40 & 1.15 & 49.7 \\
\hline 15 & Ibid & 152 & 305 & 33.7 & - & 0.38 & 1,577 & 105 & 1.24 & 0.87 & 49.4 \\
\hline 16 & Ibid & 152 & 305 & 33.7 & - & 0.76 & 1,577 & 105 & 1.65 & 0.91 & 64.6 \\
\hline 17 & Ibid & 152 & 305 & 33.7 & - & 0.76 & 1,577 & 105 & 2.25 & 1.00 & 75.2 \\
\hline 18 & Ibid & 152 & 305 & 33.7 & - & 0.76 & 1,577 & 105 & 2.16 & 1.00 & 71.8 \\
\hline 19 & Ibid & 152 & 305 & 33.7 & - & 1.14 & 1,577 & 105 & 2.45 & 0.82 & 82.9 \\
\hline 20 & Ibid & 152 & 305 & 33.7 & - & 1.14 & 1,577 & 105 & 3.03 & 0.90 & 95.4 \\
\hline 21 & Ibid & 152 & 305 & 43.8 & - & 0.38 & 1,577 & 105 & 0.98 & 0.81 & 54.8 \\
\hline 22 & Ibid & 152 & 305 & 43.8 & - & 0.38 & 1,577 & 105 & 0.47 & 0.76 & 52.1 \\
\hline 23 & Ibid & 152 & 305 & 43.8 & - & 0.38 & 1,577 & 105 & 0.37 & 0.28 & 48.7 \\
\hline 24 & Ibid & 152 & 305 & 43.8 & - & 0.76 & 1,577 & 105 & 1.57 & 0.92 & 84.0 \\
\hline 25 & Ibid & 152 & 305 & 43.8 & - & 0.76 & 1,577 & 105 & 1.37 & 1.00 & 79.2 \\
\hline 26 & Ibid & 152 & 305 & 43.8 & - & 0.76 & 1,577 & 105 & 1.66 & 1.01 & 85.0 \\
\hline 27 & Ibid & 152 & 305 & 43.8 & - & 1.14 & 1,577 & 105 & 1.74 & 0.79 & 96.5 \\
\hline 28 & Ibid & 152 & 305 & 43.8 & - & 1.14 & 1,577 & 105 & 1.68 & 0.71 & 92.6 \\
\hline 29 & Ibid & 152 & 305 & 43.8 & - & 1.14 & 1,577 & 105 & 1.75 & 0.84 & 94.0 \\
\hline 30 & Ibid & 152 & 305 & 55.2 & - & 0.38 & 1,577 & 105 & 0.69 & 0.70 & 57.9 \\
\hline 31 & Ibid & 152 & 305 & 55.2 & - & 0.38 & 1,577 & 105 & 0.48 & 0.62 & 62.9 \\
\hline 32 & Ibid & 152 & 305 & 55.2 & - & 0.38 & 1,577 & 105 & 0.49 & 0.19 & 58.1 \\
\hline 33 & Ibid & 152 & 305 & 55.2 & - & 0.76 & 1,577 & 105 & 1.21 & 0.74 & 74.6 \\
\hline 34 & Ibid & 152 & 305 & 55.2 & - & 0.76 & 1,577 & 105 & 0.81 & 0.83 & 77.6 \\
\hline 35 & Ibid & 152 & 305 & 55.2 & - & 1.14 & 1,577 & 105 & 1.43 & 0.76 & 106.5 \\
\hline 36 & Ibid & 152 & 305 & 55.2 & - & 1.14 & 1,577 & 105 & 1.45 & 0.85 & 108.0 \\
\hline 37 & Ibid & 152 & 305 & 55.2 & - & 1.14 & 1,577 & 105 & 1.18 & 0.70 & 103.3 \\
\hline 38 & De Lorenzis et al. (2002) & 120 & 240 & 43 & - & 0.30 & 1,028 & 91 & 1.16 & 0.70 & 58.5 \\
\hline 39 & Ibid & 120 & 240 & 43 & - & 0.30 & 1,028 & 91 & 0.95 & 0.80 & 65.6 \\
\hline 40 & Ibid & 150 & 300 & 38 & - & 0.45 & 1,028 & 91 & 0.95 & 0.80 & 62.0 \\
\hline 41 & Ibid & 150 & 300 & 38 & - & 0.45 & 1,028 & 91 & 1.35 & 0.80 & 67.3 \\
\hline 42 & Jiang and Teng (2007) & 152 & 305 & 38 & 0.22 & 0.68 & 3,772 & 241 & 2.55 & 0.98 & 110.1 \\
\hline 43 & Ibid & 152 & 305 & 38 & 0.22 & 0.68 & 3,772 & 241 & 2.61 & 0.97 & 107.4 \\
\hline 44 & Ibid & 152 & 305 & 38 & 0.22 & 1.02 & 3,772 & 241 & 2.79 & 0.89 & 129.0 \\
\hline 45 & Ibid & 152 & 305 & 38 & 0.22 & 1.02 & 3,772 & 241 & 3.08 & 0.93 & 135.7 \\
\hline 46 & Ibid & 152 & 305 & 38 & 0.22 & 1.36 & 3,772 & 241 & 3.70 & 0.87 & 161.3 \\
\hline 47 & Ibid & 152 & 305 & 38 & 0.22 & 1.36 & 3,772 & 241 & 3.54 & 0.88 & 158.5 \\
\hline 48 & Ibid & 152 & 305 & 37.7 & 0.28 & 0.11 & 4,332 & 260 & 0.90 & 0.94 & 48.5 \\
\hline 49 & Ibid & 152 & 305 & 37.7 & 0.28 & 0.11 & 4,332 & 260 & 0.91 & 1.09 & 50.3 \\
\hline 50 & Ibid & 152 & 305 & 42.2 & 0.26 & 0.11 & 4,332 & 260 & 0.69 & 0.73 & 48.1 \\
\hline 51 & Ibid & 152 & 305 & 42.2 & 0.26 & 0.11 & 4,332 & 260 & 0.89 & 0.97 & 51.1 \\
\hline 52 & Ibid & 152 & 305 & 42.2 & 0.26 & 0.22 & 4,332 & 260 & 1.30 & 1.18 & 65.7 \\
\hline 53 & Ibid & 152 & 305 & 42.2 & 0.26 & 0.22 & 4,332 & 260 & 1.03 & 0.94 & 62.9 \\
\hline 54 & Ibid & 152 & 305 & 47.6 & 0.28 & 0.33 & 4,332 & 251 & 1.30 & 0.90 & 82.7 \\
\hline 55 & Ibid & 152 & 305 & 47.6 & 0.28 & 0.33 & 4,332 & 251 & 1.94 & 1.13 & 85.5 \\
\hline 56 & Ibid & 152 & 305 & 47.6 & 0.28 & 0.33 & 4,332 & 251 & 1.82 & 1.06 & 85.5 \\
\hline 57 & Lam et al. (2006) & 152 & 304 & 41.1 & 0.26 & 0.17 & 3,795 & 251 & 0.90 & 0.81 & 52.6 \\
\hline 58 & Ibid & 152.5 & 305 & 41.1 & 0.26 & 0.17 & 3,795 & 251 & 1.21 & 1.08 & 57.0 \\
\hline 59 & Ibid & 152.5 & 305 & 41.1 & 0.26 & 0.17 & 3,795 & 251 & 1.11 & 1.07 & 55.4 \\
\hline 60 & Ibid & 152.5 & 305 & 38.9 & 0.25 & 0.33 & 3,795 & 251 & 1.91 & 1.06 & 76.8 \\
\hline 61 & Ibid & 152.5 & 305 & 38.9 & 0.25 & 0.33 & 3,795 & 251 & 2.08 & 1.13 & 79.1 \\
\hline 62 & Saenz and Pantelides (2006) & 152 & 304 & 41.8 & - & 0.60 & 1,220 & 87 & 1.18 & 0.92 & 83.7 \\
\hline 63 & Ibid & 152 & 304 & 47.5 & - & 0.60 & 1,220 & 87 & 0.88 & 0.93 & 81.5 \\
\hline 64 & Ibid & 152 & 304 & 40.3 & - & 1.20 & 1,220 & 87 & 2.04 & 0.92 & 108.1 \\
\hline 65 & Ibid & 152 & 304 & 41.7 & - & 1.20 & 1,220 & 87 & 1.76 & 1.08 & 109.5 \\
\hline 66 & Valdmanis et al. (2007) & 150 & 300 & 40 & 0.17 & 0.17 & 1,906 & 201 & 0.63 & 0.89 & 66.0 \\
\hline
\end{tabular}


Table 1. (Continued.)

\begin{tabular}{|c|c|c|c|c|c|c|c|c|c|c|c|}
\hline Number & Source of data & $\begin{array}{c}d \\
(\mathrm{~mm})\end{array}$ & $\begin{array}{c}h \\
(\mathrm{~mm})\end{array}$ & $\begin{array}{c}f_{c o}^{\prime} \\
(\mathrm{MPa})\end{array}$ & $\begin{array}{l}\varepsilon_{c o} \\
(\%)\end{array}$ & $\begin{array}{c}t \\
(\mathrm{~mm})\end{array}$ & $\begin{array}{c}f_{f} \\
(\mathrm{MPa})\end{array}$ & $\begin{array}{c}E_{f} \\
(\mathrm{GPa})\end{array}$ & $\begin{array}{l}\varepsilon_{c c} \\
(\%)\end{array}$ & $\begin{array}{l}\varepsilon_{f e} \\
(\%)\end{array}$ & $\begin{array}{c}f_{c c} \\
(\mathrm{MPa})\end{array}$ \\
\hline 67 & Ibid & 150 & 300 & 40 & 0.17 & 0.34 & 2,389 & 231 & 1.07 & 0.84 & 87.2 \\
\hline 68 & Ibid & 150 & 300 & 40 & 0.17 & 0.51 & 2,661 & 236 & 1.36 & 0.69 & 96.0 \\
\hline 69 & Ibid & 150 & 300 & 44.3 & 0.17 & 0.17 & 1,906 & 201 & 0.58 & 0.74 & 73.3 \\
\hline 70 & Ibid & 150 & 300 & 44.3 & 0.17 & 0.34 & 2,389 & 231 & 0.54 & 0.43 & 82.6 \\
\hline 71 & Ibid & 150 & 300 & 44.3 & 0.17 & 0.51 & 2,661 & 236 & 0.94 & 0.78 & 115.1 \\
\hline 72 & Berthet et al. (2005) & 160 & 320 & 25 & 0.23 & 0.17 & 3,200 & 230 & 1.63 & 0.96 & 42.8 \\
\hline 73 & Ibid & 160 & 320 & 25 & 0.23 & 0.17 & 3,200 & 230 & 0.93 & 0.96 & 37.8 \\
\hline 74 & Ibid & 160 & 320 & 25 & 0.23 & 0.17 & 3,200 & 230 & 1.67 & 0.96 & 45.8 \\
\hline 75 & Ibid & 160 & 320 & 25 & 0.23 & 0.33 & 3,200 & 230 & 1.73 & 0.90 & 56.7 \\
\hline 76 & Ibid & 160 & 320 & 25 & 0.23 & 0.33 & 3,200 & 230 & 1.58 & 0.91 & 55.2 \\
\hline 77 & Ibid & 160 & 320 & 25 & 0.23 & 0.33 & 3,200 & 230 & 1.68 & 0.91 & 56.1 \\
\hline 78 & Ibid & 160 & 320 & 40.1 & 0.2 & 0.11 & 3,200 & 230 & 0.55 & 1.02 & 49.8 \\
\hline 79 & Ibid & 160 & 320 & 40.1 & 0.2 & 0.11 & 3,200 & 230 & 0.66 & 0.95 & 50.8 \\
\hline 80 & Ibid & 160 & 320 & 40.1 & 0.2 & 0.11 & 3,200 & 230 & 0.61 & 1.20 & 48.8 \\
\hline 81 & Ibid & 160 & 320 & 40.1 & 0.2 & 0.17 & 3,200 & 230 & 0.66 & 0.88 & 53.7 \\
\hline 82 & Ibid & 160 & 320 & 40.1 & 0.2 & 0.17 & 3,200 & 230 & 0.62 & 0.85 & 54.7 \\
\hline 83 & Ibid & 160 & 320 & 40.1 & 0.2 & 0.17 & 3,200 & 230 & 0.64 & 1.04 & 51.8 \\
\hline 84 & Ibid & 160 & 320 & 40.1 & 0.2 & 0.22 & 3,200 & 230 & 0.60 & 0.79 & 59.7 \\
\hline 85 & Ibid & 160 & 320 & 40.1 & 0.2 & 0.22 & 3,200 & 230 & 0.69 & 0.83 & 60.7 \\
\hline 86 & Ibid & 160 & 320 & 40.1 & 0.2 & 0.22 & 3,200 & 230 & 0.73 & 0.81 & 60.2 \\
\hline 87 & Ibid & 160 & 320 & 40.1 & 0.2 & 0.44 & 3,200 & 230 & 1.44 & 0.92 & 91.6 \\
\hline 88 & Ibid & 160 & 320 & 40.1 & 0.20 & 0.44 & 3,200 & 230 & 1.36 & 0.97 & 89.6 \\
\hline 89 & Ibid & 160 & 320 & 40.1 & 0.20 & 0.44 & 3,200 & 230 & 1.17 & 0.89 & 86.6 \\
\hline 90 & Ibid & 160 & 320 & 40.1 & 0.20 & 0.99 & 3,200 & 230 & 2.46 & 0.99 & 142.4 \\
\hline 91 & Ibid & 160 & 320 & 40.1 & 0.20 & 0.99 & 3,200 & 230 & 2.39 & 1.00 & 140.4 \\
\hline 92 & Ibid & 160 & 320 & 40.1 & 0.20 & 1.32 & 3,200 & 230 & 2.70 & 1.00 & 166.3 \\
\hline 93 & Ibid & 160 & 320 & 52 & 0.23 & 0.33 & 3,200 & 230 & 0.83 & 0.93 & 82.6 \\
\hline 94 & Ibid & 160 & 320 & 52 & 0.23 & 0.33 & 3,200 & 230 & 0.70 & 0.87 & 82.8 \\
\hline 95 & Ibid & 160 & 320 & 52 & 0.23 & 0.33 & 3,200 & 230 & 0.77 & 0.89 & 82.3 \\
\hline 96 & Ibid & 160 & 320 & 52 & 0.23 & 0.66 & 3,200 & 230 & 1.14 & 0.67 & 108.1 \\
\hline 97 & Ibid & 160 & 320 & 52 & 0.23 & 0.66 & 3,200 & 230 & 1.12 & 0.87 & 112 \\
\hline 98 & Ibid & 160 & 320 & 52 & 0.23 & 0.66 & 3,200 & 230 & 1.12 & 0.882 & 107.9 \\
\hline
\end{tabular}

For square sections, the energy absorbed by CFRP (Fig. 4) was modified by adding the corner energy ratio $k_{c}$

$$
U_{f}=\sqrt{k_{c}} \rho_{f}\left(0.5 f_{f e} \varepsilon_{f e}\right)
$$

Early studies on steel confined concrete have reported the wellknown assumption that the concrete in a square section is confined by the transverse reinforcement through arching actions (Mander et al. 1988; Cusson and Paultre 1995). Consequently, only the concrete contained by four second-degree parabolas [Fig. 3(a)] is well-confined, whereas the confinement effect at other zones is negligible. As further evidence, a few experimental (Mirmiran et al. 1998; Rochette and Labossiére 2000) and analytical (Karabinis et al. 2008) studies also confirmed that only part of the section is fully confined in terms of FRP-confined concrete columns. The writers assumed that the energy absorption of the effective area is proportional to the total energy absorbed of the entire section [Eq. (3)]. In this paper, the energy absorption of square specimens is assumed as the energy absorbed by the effective area only. This energy can be calculated by combining Eq. (3) and the shape factor $k_{s}$, as introduced by ACI 440.2R-08 (ACI 2008)

$$
\begin{gathered}
U_{c c}=k_{s} \frac{\left(\varepsilon_{c c}-\varepsilon_{c o}\right)\left(f_{c c}^{\prime}+f_{c o}^{\prime}\right)}{2} \\
k_{s}=1-\frac{2(b-2 r)^{2}}{3\left[b^{2}-r^{2}(4-\pi)\right]}
\end{gathered}
$$

The same methodology used in establishing the expression for circular sections was utilized for square columns. Fig. 4 and Eq. (17) show the relationship of the energy absorption in this case

$$
U_{c c}=8.3 U_{f}
$$

Substituting Eqs. (14) and (15) into Eq. (17) results in Eq. (18)

$$
\varepsilon_{c c}=\varepsilon_{c o}+\frac{t k \sqrt{k_{c}} \Psi f_{f e} \varepsilon_{f e}}{k_{s}\left(f_{c c}^{\prime}+f_{c o}^{\prime}\right)}
$$

where the proportion factor $k=8.3$. Eq. (18) can be used to calculate the strain of CFRP-confined concrete columns in square sections. The Lam and Teng (2003b) model was adopted to express another form of Eq. (18)

$$
\varepsilon_{c c}=\varepsilon_{c o}+\frac{t k \sqrt{k_{c}} \Psi f_{f e} \varepsilon_{f e}}{k_{s}\left(2 f_{c o}^{\prime}+3.3 k_{s} f_{l}\right)}
$$

where $f_{l}=$ equivalent confining pressure of a square section, which can be estimated (Lam and Teng 2003b) as

$$
f_{l}=\frac{\sqrt{2} t f_{f e}}{b}
$$


Table 2. Database of Square Specimens

\begin{tabular}{|c|c|c|c|c|c|c|c|c|c|c|c|}
\hline Number & Source of data & $\begin{array}{c}b \\
(\mathrm{~mm})\end{array}$ & $\begin{array}{c}h \\
(\mathrm{~mm})\end{array}$ & $\begin{array}{c}r \\
(\mathrm{~mm})\end{array}$ & $\begin{array}{c}f_{c o}^{\prime} \\
(\mathrm{MPa})\end{array}$ & $\begin{array}{c}t \\
(\mathrm{~mm})\end{array}$ & $\begin{array}{c}f_{f} \\
\text { (MPa) }\end{array}$ & $\begin{array}{c}E_{f} \\
(\mathrm{GPa})\end{array}$ & $\begin{array}{l}\varepsilon_{c c} \\
(\%)\end{array}$ & $\begin{array}{l}\varepsilon_{f e} \\
(\%)\end{array}$ & $\begin{array}{c}f_{c c}^{\prime} \\
(\mathrm{MPa})\end{array}$ \\
\hline 1 & Rochette and Labossière (2000) & 152 & 500 & 25 & 42 & 0.90 & 1,265 & 83 & 0.94 & 0.56 & 50.9 \\
\hline 2 & Ibid & 152 & 500 & 25 & 42 & 0.90 & 1,265 & 83 & 0.89 & 0.63 & 52.0 \\
\hline 3 & Ibid & 152 & 500 & 38 & 42 & 0.90 & 1,265 & 83 & 1.08 & 0.71 & 55.4 \\
\hline 4 & Ibid & 152 & 500 & 25 & 44 & 1.20 & 1,265 & 83 & 1.35 & 0.59 & 56.4 \\
\hline 5 & Ibid & 152 & 500 & 25 & 36 & 1.20 & 1,265 & 83 & 2.04 & 0.70 & 50.6 \\
\hline 6 & Ibid & 152 & 500 & 25 & 36 & 1.50 & 1,265 & 83 & 2.12 & 0.65 & 52.9 \\
\hline 7 & Ibid & 152 & 500 & 38 & 36 & 1.20 & 1,265 & 83 & 1.92 & 0.89 & 58.1 \\
\hline 8 & Ibid & 152 & 500 & 38 & 36 & 1.50 & 1,265 & 83 & 2.39 & 0.86 & 62.8 \\
\hline 9 & Lam and Teng (2003b) & 150 & 600 & 25 & 34 & 0.17 & 1,577 & 105 & 0.96 & 1.05 & 37.6 \\
\hline 10 & Ibid & 150 & 600 & 15 & 34 & 0.33 & 1,577 & 105 & 0.87 & 0.97 & 39.7 \\
\hline 11 & Ibid & 150 & 600 & 25 & 34 & 0.33 & 1,577 & 105 & 0.85 & 1.08 & 41.8 \\
\hline 12 & Ibid & 150 & 600 & 15 & 24 & 0.50 & 1,577 & 105 & 1.80 & 0.87 & 32.0 \\
\hline 13 & Ibid & 150 & 600 & 25 & 24 & 0.50 & 1,577 & 105 & 1.52 & 1.16 & 37.1 \\
\hline 14 & Pessiki et al. (2001) & 152 & 610 & 38 & 26 & 1.00 & 580 & 38 & 1.50 & 0.83 & 34.4 \\
\hline 15 & Ibid & 152 & 610 & 38 & 26 & 2.00 & 580 & 38 & 1.90 & 0.90 & 43.7 \\
\hline 16 & Chaallal et al. (2003) & 133 & 305 & 25 & 21 & 0.12 & 3,650 & 231 & 0.40 & 0.40 & 23.6 \\
\hline 17 & Ibid & 133 & 305 & 25 & 21 & 0.24 & 3,650 & 231 & 0.40 & 0.50 & 27.8 \\
\hline 18 & Ibid & 133 & 305 & 25 & 21 & 0.36 & 3,650 & 231 & 0.60 & 0.52 & 31.8 \\
\hline 19 & Ibid & 133 & 305 & 25 & 21 & 0.48 & 3,650 & 231 & 0.70 & 0.53 & 35.8 \\
\hline 20 & Ibid & 133 & 305 & 25 & 41 & 0.12 & 3,650 & 231 & 0.30 & 0.08 & 42.0 \\
\hline 21 & Ibid & 133 & 305 & 25 & 41 & 0.24 & 3,650 & 231 & 0.30 & 0.11 & 43.0 \\
\hline 22 & Ibid & 133 & 305 & 25 & 41 & 0.36 & 3,650 & 231 & 0.40 & 0.15 & 44.6 \\
\hline 23 & Ibid & 133 & 305 & 25 & 41 & 0.48 & 3,650 & 231 & 0.40 & 0.20 & 47.1 \\
\hline 24 & Ibid & 150 & 300 & 15 & 33 & 0.33 & 4,364 & 219 & 1.22 & - & 46.9 \\
\hline 25 & Ibid & 150 & 300 & 15 & 32 & 0.33 & 4,364 & 219 & 1.22 & - & 46.2 \\
\hline 26 & Ibid & 150 & 300 & 15 & 31 & 0.33 & 4,364 & 219 & 1.22 & - & 44.7 \\
\hline 27 & Ibid & 150 & 300 & 30 & 33 & 0.17 & 4,364 & 219 & 1.34 & - & 41.9 \\
\hline 28 & Ibid & 150 & 300 & 30 & 31 & 0.17 & 4,364 & 219 & 1.34 & - & 40.4 \\
\hline 29 & Ibid & 150 & 300 & 30 & 33 & 0.17 & 4,364 & 219 & 1.34 & - & 42.4 \\
\hline 30 & Ibid & 150 & 300 & 30 & 33 & 0.33 & 4,364 & 219 & 1.44 & - & 51.1 \\
\hline 31 & Ibid & 150 & 300 & 30 & 31 & 0.33 & 4,364 & 219 & 1.44 & - & 49.6 \\
\hline 32 & Ibid & 150 & 300 & 30 & 33 & 0.33 & 4,364 & 219 & 1.44 & - & 51.6 \\
\hline 33 & Ibid & 150 & 300 & 45 & 30 & 0.17 & 4,364 & 219 & 1.39 & - & 41.0 \\
\hline 34 & Ibid & 150 & 300 & 45 & 33 & 0.17 & 4,364 & 219 & 1.39 & - & 43.5 \\
\hline 35 & Ibid & 150 & 300 & 45 & 29 & 0.17 & 4,364 & 219 & 1.39 & - & 40.2 \\
\hline 36 & Ibid & 150 & 300 & 45 & 30 & 0.33 & 4,364 & 219 & 1.57 & - & 51.9 \\
\hline 37 & Ibid & 150 & 300 & 45 & 33 & 0.33 & 4,364 & 219 & 1.57 & - & 54.4 \\
\hline 38 & Ibid & 150 & 300 & 45 & 29 & 0.33 & 4,364 & 219 & 1.57 & - & 51.1 \\
\hline 39 & Ibid & 150 & 300 & 60 & 31 & 0.17 & 4,364 & 219 & 1.65 & - & 42.8 \\
\hline 40 & Ibid & 150 & 300 & 60 & 31 & 0.17 & 4,364 & 219 & 1.65 & - & 43.0 \\
\hline 41 & Ibid & 150 & 300 & 60 & 34 & 0.17 & 4,364 & 219 & 1.65 & - & 45.4 \\
\hline 42 & Ibid & 150 & 300 & 60 & 31 & 0.33 & 4,364 & 219 & 1.76 & - & 54.8 \\
\hline 43 & Ibid & 150 & 300 & 60 & 31 & 0.33 & 4,364 & 219 & 1.76 & - & 55.0 \\
\hline 44 & Ibid & 150 & 300 & 60 & 34 & 0.33 & 4,364 & 219 & 1.76 & - & 57.4 \\
\hline 45 & Ibid & 150 & 300 & 30 & 54 & 0.33 & 3,788 & 226 & 1.37 & - & 69.6 \\
\hline 46 & Ibid & 150 & 300 & 30 & 53 & 0.33 & 3,788 & 226 & 1.37 & - & 69.2 \\
\hline 47 & Ibid & 150 & 300 & 30 & 49 & 0.33 & 3,788 & 226 & 1.37 & - & 65.5 \\
\hline 48 & Ibid & 150 & 300 & 45 & 53 & 0.17 & 3,788 & 226 & 1.51 & - & 62.7 \\
\hline 49 & Ibid & 150 & 300 & 45 & 52 & 0.17 & 3,788 & 226 & 1.51 & - & 61.0 \\
\hline 50 & Ibid & 150 & 300 & 45 & 53 & 0.17 & 3,788 & 226 & 1.51 & - & 62.8 \\
\hline 51 & Ibid & 150 & 300 & 45 & 53 & 0.33 & 3,788 & 226 & 1.65 & - & 72.1 \\
\hline 52 & Ibid & 150 & 300 & 45 & 52 & 0.33 & 3,788 & 226 & 1.65 & - & 70.4 \\
\hline 53 & Ibid & 150 & 300 & 45 & 53 & 0.33 & 3,788 & 226 & 1.65 & - & 72.2 \\
\hline 54 & Ibid & 150 & 300 & 60 & 54 & 0.17 & 3,788 & 226 & 1.28 & - & 64.3 \\
\hline 55 & Ibid & 150 & 300 & 60 & 52 & 0.17 & 3,788 & 226 & 1.28 & - & 62.4 \\
\hline 56 & Ibid & 150 & 300 & 60 & 52 & 0.17 & 3,788 & 226 & 1.28 & - & 62.7 \\
\hline 57 & Ibid & 150 & 300 & 60 & 54 & 0.33 & 3,788 & 226 & 1.37 & - & 74.6 \\
\hline 58 & Ibid & 150 & 300 & 60 & 52 & 0.33 & 3,788 & 226 & 1.37 & - & 72.7 \\
\hline 59 & Ibid & 150 & 300 & 60 & 52 & 0.33 & 3,788 & 226 & 1.37 & - & 73.0 \\
\hline 60 & Tao et al. (2008) & 150 & 450 & 20 & 22 & 0.17 & 4,470 & 239 & 2.53 & - & 30.3 \\
\hline 61 & Ibid & 150 & 450 & 20 & 22 & 0.34 & 4,470 & 239 & 3.95 & - & 38.5 \\
\hline 62 & Ibid & 150 & 450 & 20 & 20 & 0.34 & 4,470 & 239 & 3.34 & - & 36.0 \\
\hline 63 & Ibid & 150 & 450 & 35 & 22 & 0.34 & 4,470 & 239 & 3.66 & - & 42.8 \\
\hline 64 & Ibid & 150 & 450 & 35 & 20 & 0.34 & 4,470 & 239 & 3.48 & - & 40.3 \\
\hline 65 & Ibid & 150 & 450 & 50 & 22 & 0.34 & 4,470 & 239 & 3.87 & - & 45.9 \\
\hline 66 & Ibid & 150 & 450 & 50 & 20 & 0.34 & 4,470 & 239 & 3.43 & - & 43.4 \\
\hline
\end{tabular}




\begin{tabular}{|c|c|c|c|c|c|c|c|c|c|c|c|}
\hline Number & Source of data & $\begin{array}{c}b \\
(\mathrm{~mm})\end{array}$ & $\begin{array}{c}h \\
(\mathrm{~mm})\end{array}$ & $\begin{array}{c}r \\
(\mathrm{~mm})\end{array}$ & $\begin{array}{c}f_{c o}^{\prime} \\
(\mathrm{MPa})\end{array}$ & $\begin{array}{c}t \\
(\mathrm{~mm})\end{array}$ & $\begin{array}{c}f_{f} \\
(\mathrm{MPa})\end{array}$ & $\begin{array}{c}E_{f} \\
(\mathrm{GPa})\end{array}$ & $\begin{array}{l}\varepsilon_{c c} \\
(\%)\end{array}$ & $\begin{array}{l}\varepsilon_{f e} \\
(\%)\end{array}$ & $\begin{array}{c}f_{c c}^{\prime} \\
(\mathrm{MPa})\end{array}$ \\
\hline 67 & Ibid & 150 & 450 & 20 & 50 & 0.34 & 4,200 & 241 & 1.66 & - & 65.0 \\
\hline 68 & Ibid & 150 & 450 & 35 & 50 & 0.34 & 4,200 & 241 & 2.08 & - & 69.1 \\
\hline 69 & Ibid & 150 & 450 & 50 & 50 & 0.34 & 4,200 & 241 & 1.65 & - & 71.9 \\
\hline
\end{tabular}

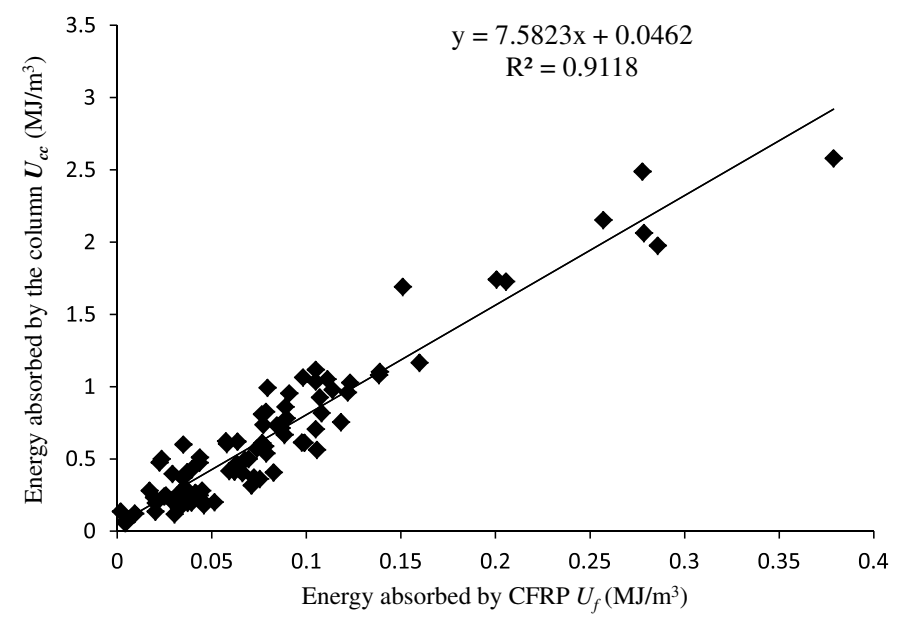

Fig. 2. Energy relationship of circular sections

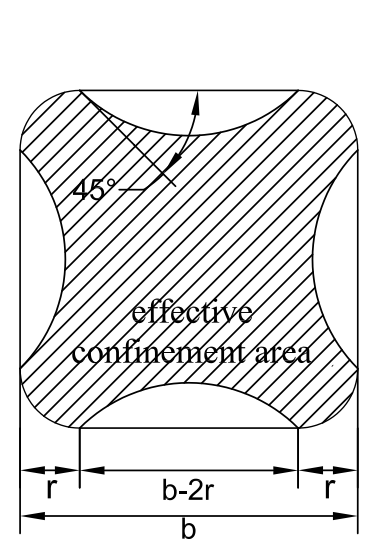

(a)

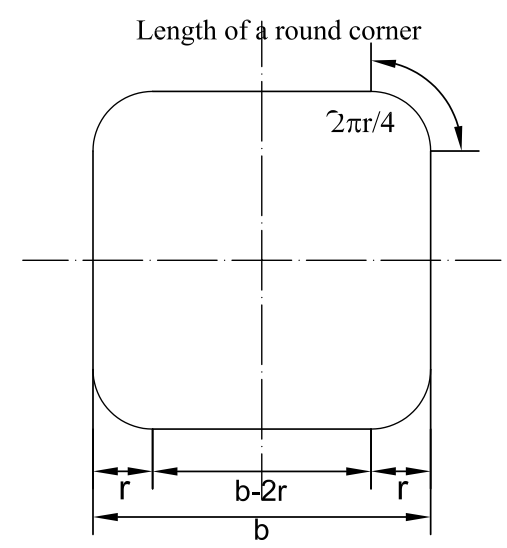

(b)

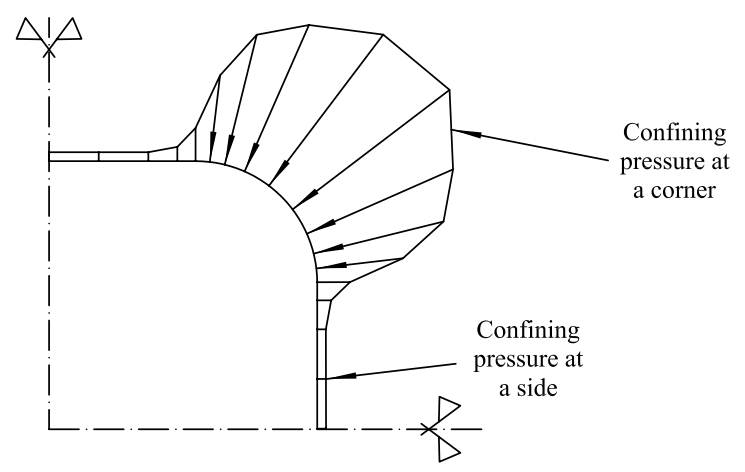

(c)

Fig. 3. (a) Effective confinement area; (b) confining pressure of square sections; (c) round corners of square sections

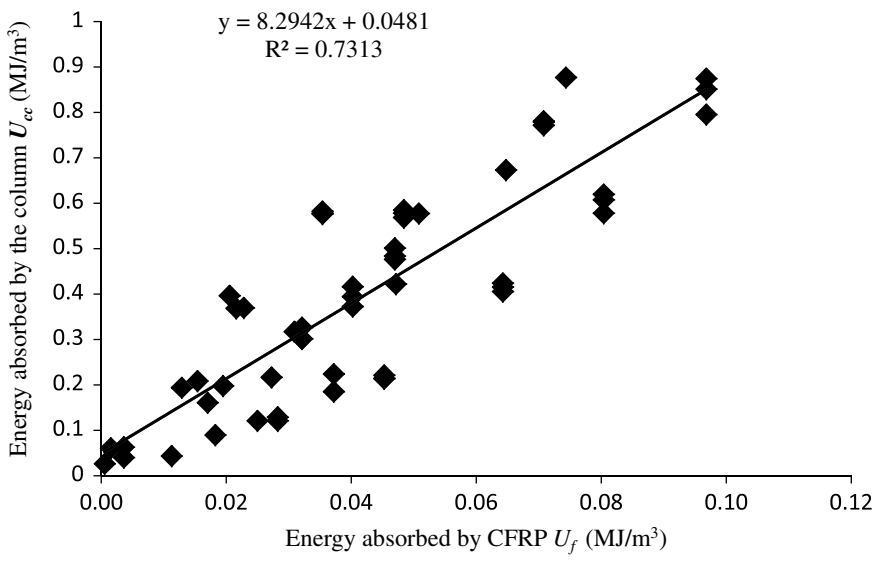

Fig. 4. Energy relationship of square sections

\section{Verification of the Proposed Model}

\section{Statistical Methods of Verification}

In the writers' paper, the model performance was tested by using two statistical indicators, as follows: (1) mean square error (MSE), and (2) average absolute error (AAE), as determined by Eqs. (21) and (22):

$$
\begin{aligned}
\text { MSE } & =\frac{\sum_{1}^{n}\left(\frac{\operatorname{pre}_{i}-\exp _{i}}{\exp _{i}}\right)^{2}}{N} \\
\mathrm{AAE} & =\frac{\sum_{1}^{n}\left|\frac{\operatorname{pre}_{i}-\exp _{i}}{\exp _{i}}\right|}{N}
\end{aligned}
$$

where pre $=$ model predictions; $\exp =$ experimental results; and $N=$ total number of test data. In general, the mean square error shows the errors to be more significant compared with the average absolute error.

\section{Circular FRP-Confined Concrete Columns}

A total of 98 data points were plotted (Fig. 5) to assess the performance of existing models and the proposed model. Seven existing models were considered in this verification [Karbhari and Gao 1997; Toutanji 1999; De Lorenzis and Tepfers 2003; ACI 440.2R-08 (ACI 2008); Teng et al. 2009; Rousakis et al. 2012; Yazici and Hadi 2012]. Because of the limited space in the writers' paper, only four models that have comparable performance are shown (Fig. 5). Fig. 6 presents all seven models to illustrate a comparison of the models' performance.

Based on the two statistical indicators, the models of ACI 440.2R-08 (ACI 2008) and Rousakis et al. (2012) provide the best 


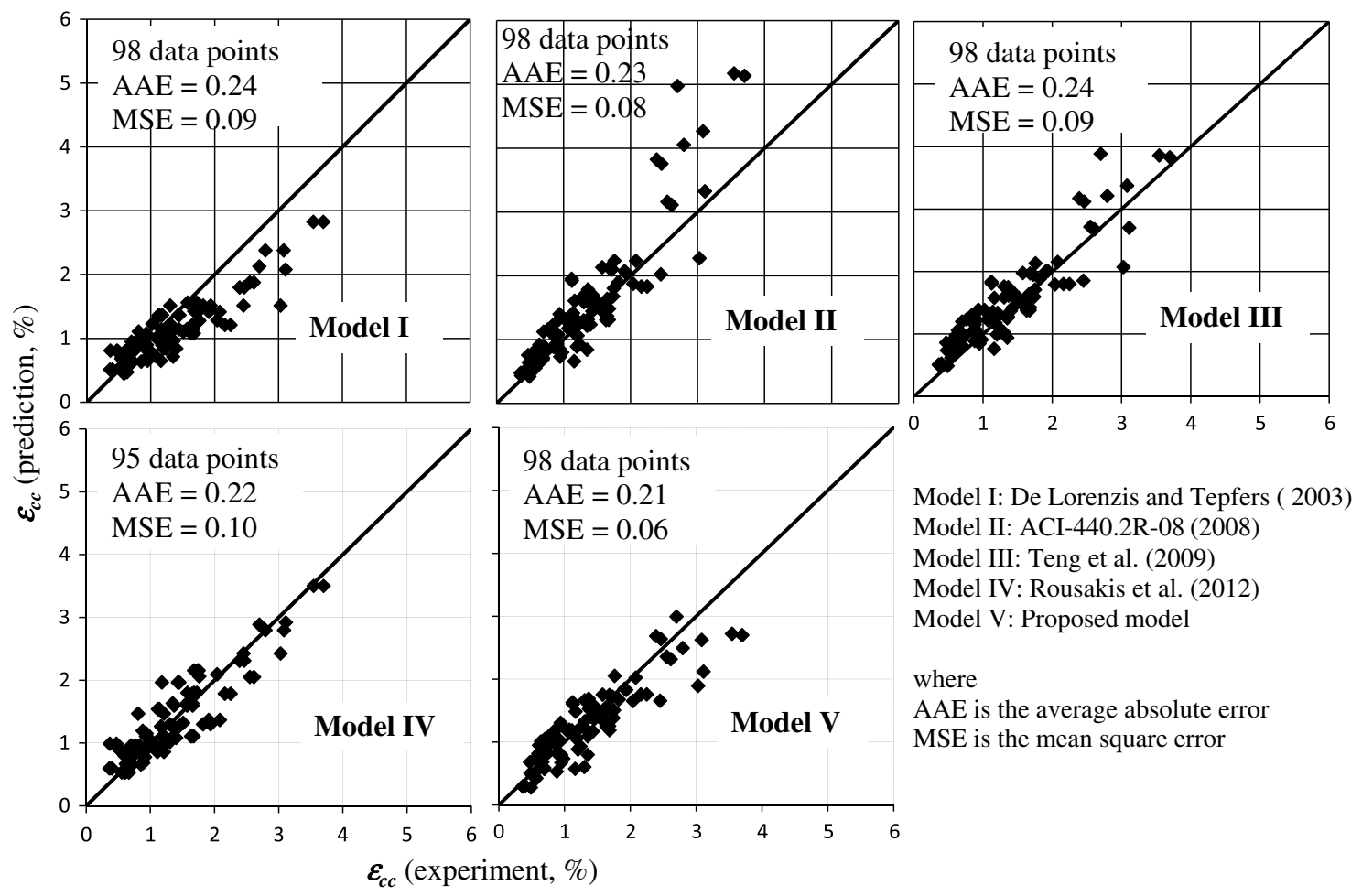

Fig. 5. Performance of models on circular specimens

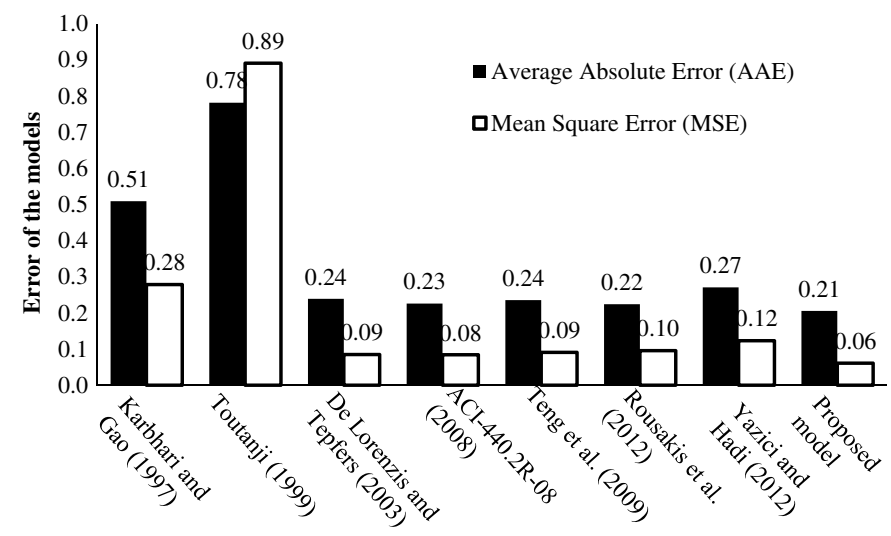

Fig. 6. Accuracy comparisons for strain prediction of circular specimens among the models

strain prediction, followed by the models of Lam and Teng (2003a) and De Lorenzis and Tepfers (2003). The model of Rousakis et al. (2012) shows good agreement with experimental results, with the exception of high-modulus (HM) CFRP such that three specimens using HM CFRP were excluded from the verification of this model. The proposed model in this paper shows slightly better estimates than the model of ACI 440.2R-08 (ACI 2008) and Rousakis et al. (2012).

The model of ACI 440.2R-08 (ACI 2008) suggests that the minimum confinement ratio $f_{l} / f_{c o}^{\prime}$ of 0.08 should be used. This minimum limit was recommended based on increasing the strength of CFRP-confined concrete. In particular, in earthquake-prone regions the ductility of a column may need to be increased, leading to a case of insufficient strength confinement, whereas the ductility enhancement still could be expected (Mirmiran et al. 1998; Wang and $\mathrm{Wu}$ 2008). Therefore, eight specimens (insufficient confinement)

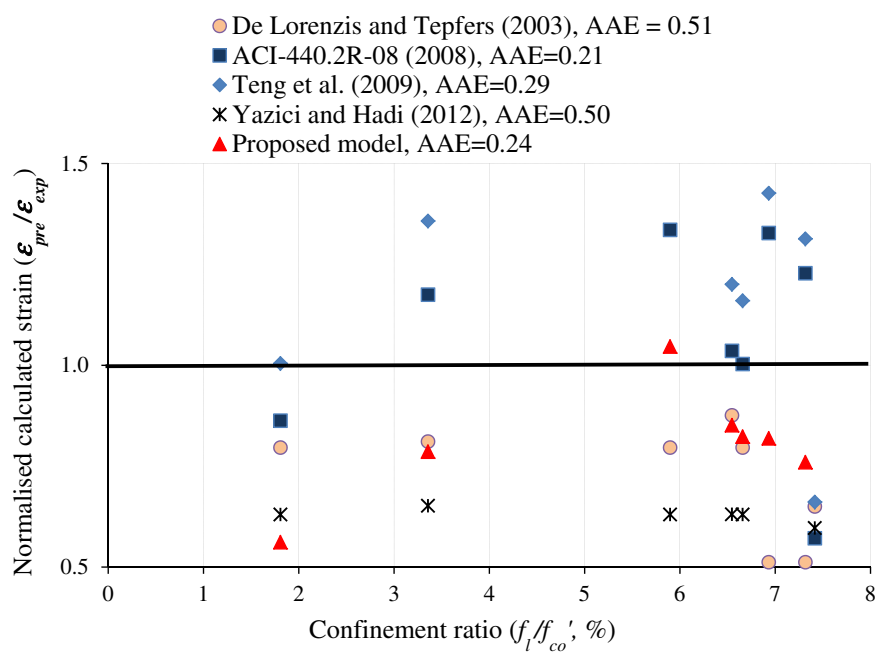

Fig. 7. Performance of models on circular specimens (insufficient confinement)

having a confinement ratio of less than 0.08 were extracted from the full database to verify the models in this case (Fig. 7). Based on the strain-estimation equations of the subsequent models, if the confinement pressure is equal to zero, the strain of confined concrete calculated by the models of ACI 440.2R-08 (ACI 2008) would be $1.5 \times$ the unconfined concrete strain. Consequently, when the confinement pressure $f_{l}$ reaches zero the strain prediction from the model of ACI 440.2R-08 (ACI 2008) will overestimate the actual strain. When that model was verified by the database, it exhibited good predictions for insufficient confined specimens. The prediction of the proposed model still shows a quite good correlation with the test data, whereas other models show scatter of the test data (Fig. 7). 


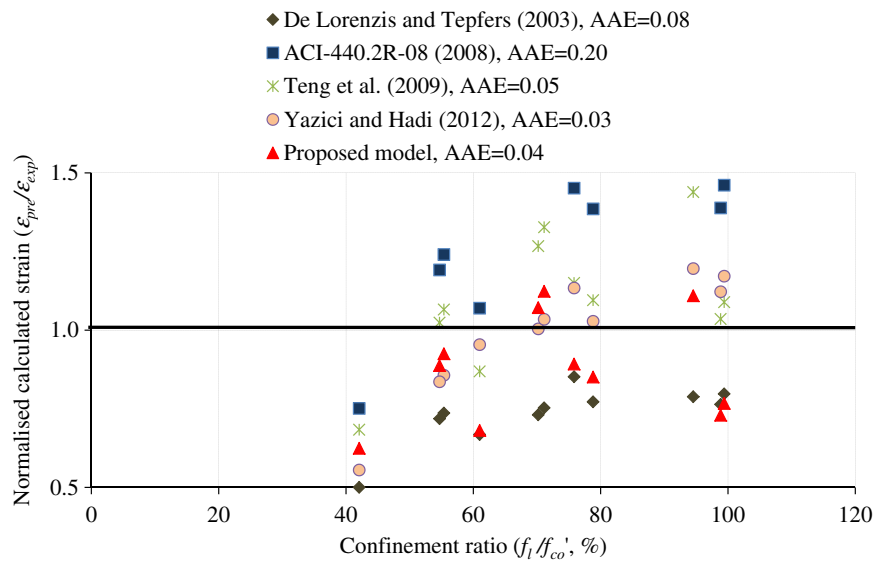

Fig. 8. Performance of models on circular specimens (heavy confinement)

In addition, the models of ACI 440.2R-08 (ACI 2008) and Teng et al. (2009) generally tend to overestimate the strain of confined concrete when the confinement ratio $\left(f_{l} / f_{c o}^{\prime}\right)$ is large. The differences between the experimental and predicted values become considerably larger when the confinement ratio was larger than $40 \%$ (Fig. 5), remarked as heavy confinement. Thus, eleven heavy confined specimens were extracted from the database to compare these models (Fig. 8). The models of ACI 440.2R-08 (ACI 2008), Teng et al. (2009), and De Lorenzis and Tepfers (2003) show that the precision of these models are not good, whereas the model of Yazici and Hadi (2012) exhibits good predictions in this case. For further verification, Fig. 8 shows a very good correlation between the predicted and actual strain of heavily confined circular sections. The average absolute error of the proposed model is $5 \times$ less than the model of ACI 440.2R-08 (ACI 2008).

In summary, the proposed model predicts very close results for the strain of CFRP-confined concrete. In addition, the proposed model also shows good agreement with the test data in the range of insufficient and heavy confinement as defined previously.

\section{Square FRP-Confined Concrete Columns}

The same procedure was carried out to verify the proposed model for square sections. A total of 69 data points (Fig. 9) were plotted to assess the performance of existing models and the proposed model. Four existing models were considered in this verification [Shehata et al. 2002; Lam and Teng 2003b; ACI 440.2R-08 (ACI 2008); Ilki et al. 2008].

Comparing the existing models for square sections, the models of Lam and Teng (2003a), ACI 440.2R-08 (ACI 2008), and Ilki et al. (2008) show quite good predictions for the strain of CFRP-confined concrete. Among these existing models, the results from the model of Ilki et al. (2008) overestimate the actual values, whereas the other models present a good general trend. However, the proposed model gives a better precision than the other models in estimating the strain of CFRP-confined concrete columns (Figs. 9 and 10).

For insufficient confined specimens (places close to the origin of the coordinates), the models of Lam and Teng (2003b), ACI 440.2R-08 (ACI 2008), and the proposed model show good predictions. The models of Ilki et al. (2008) and Shehata et al. (2002) do not exhibit close correlation in this case. In addition, all five models underestimate the strain of confined concrete when the confinement ratio is high [six data points have a measured strain greater than 3 (Fig. 9)].
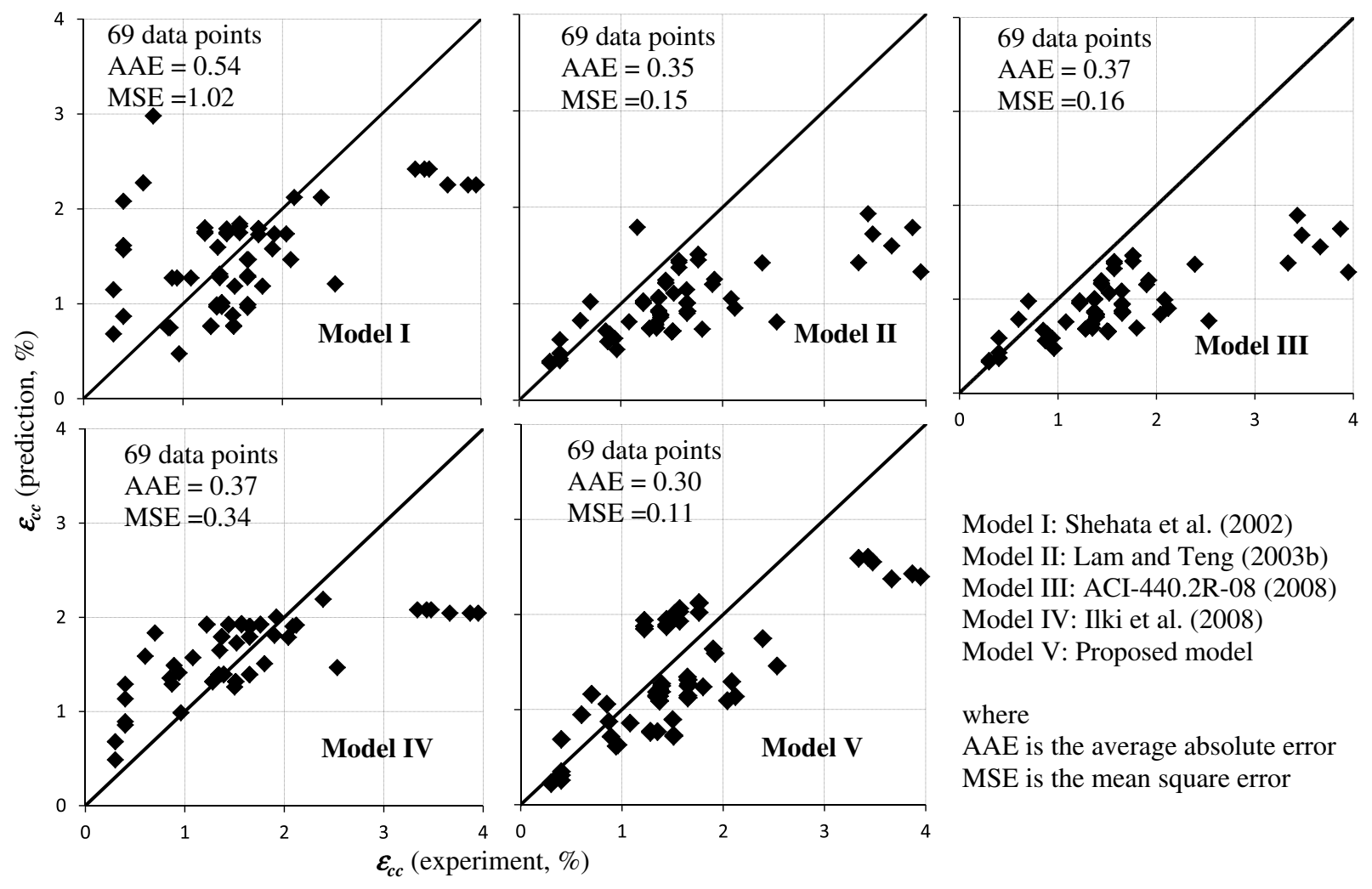

Model I: Shehata et al. (2002)

Model II: Lam and Teng (2003b)

Model III: ACI-440.2R-08 (2008)

Model IV: Ilki et al. (2008)

Model V: Proposed model

where

AAE is the average absolute error

$\mathrm{MSE}$ is the mean square error

Fig. 9. Performance of models on square specimens 


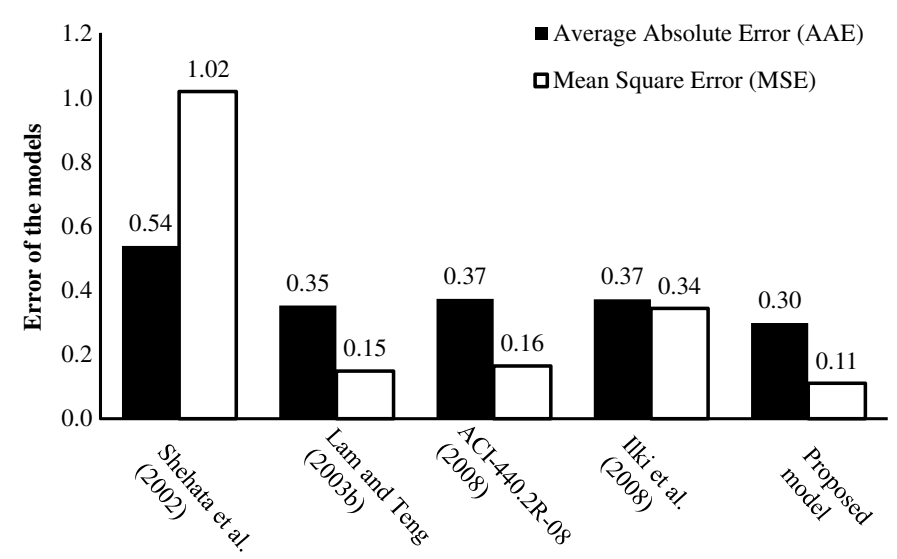

Fig. 10. Accuracy comparisons for strain prediction of square specimens among the models

\section{Conclusions}

From the theoretical analyses presented in this paper, the following conclusions are drawn:

- The proposed model provides very good predictions compared with the experimental results, and it also shows a good agreement with the test data in the range of insufficient and heavy confinement, which are usually not predicted well by other models;

- Only a proportion of the energy absorbed by the entire column is transferred to rupture the FRP; and

- The formula to calculate the strain of square sections is still not as good as that of circular sections; thus, further study needs to be carried out in this case.

Finally, a new model is proposed in this paper to calculate the strain of confined concrete based on the energy-absorption method. The performance of the proposed model shows very good correlations with experimental results. However, the precision of the proposed model should improve when it is calibrated with a larger reliable database in the future. This methodology can be developed to cover reinforced concrete columns confined with FRP.

\section{Acknowledgments}

The first writer acknowledges the Vietnamese Government and the University of Wollongong for the support of his full Ph.D. scholarship. The writers thank Ph.D. scholar Mr. Ida Bagus Rai Widiarsa for his database. Furthermore, the constructive comments of the editor and reviewers are gratefully appreciated.

\section{Notation}

The following symbols are used in this paper:

$A_{c c}=$ gross-sectional area of confined concrete;

$b=$ side length of a square section;

$d=$ diameter of the section;

$d l=$ increment of the displacement;

$d \varepsilon_{c}=$ increment of the axial strain;

$f_{c}=$ stress of concrete;

$f_{f}=$ rupture strength of FRP obtained from flat-coupon tests;

$f_{f e}=$ actual rupture strength of FRP on the columns;

$f_{l}=$ confining pressure of the confined concrete specimen;

$f_{c c}^{\prime}=$ confined concrete strength;

$f_{c o}^{\prime}=$ unconfined concrete strength; $k=$ proportion factor showing the relationship between the energy absorption of the column core and external FRP;

$k_{c}=$ corner-energy ratio;

$l=$ displacement;

$P=$ applied load;

$r=$ radius of the round corner of the section;

$t=$ thickness of FRP;

$U=$ strain energy;

$U_{c c}=$ volumetric strain energy of confined concrete;

$U_{f}=$ volumetric strain energy of FRP;

$W=$ work done by the applied load;

$W_{c c}=$ strain energy of confined concrete;

$W_{f}=$ strain energy of FRP;

$\varepsilon_{c}=$ axial strain of concrete;

$\varepsilon_{c c}=$ axial strain at the peak stress of confined concrete;

$\varepsilon_{c o}=$ axial strain at the peak stress of unconfined concrete;

$\varepsilon_{f}=$ rupture strain of FRP obtained from flat-coupon tests;

$\varepsilon_{f e}=$ rupture strain of FRP on the columns; and

$\rho_{f}=$ volumetric ratio of FRP.

\section{References}

Al-Salloum, Y. A. (2007). "Influence of edge sharpness on the strength of square concrete columns confined with FRP composite laminates." Compos. Part B Eng., 38(5), 640-650.

American Concrete Institute (ACI). (2008). "Guide for the design and construction of externally bonded FRP systems for strengthening concrete structures." ACI 440.2R-08, Farmington Hills, MI.

Berthet, J. F., Ferrier, E., and Hamelin, P. (2005). "Compressive behavior of concrete externally confined by composite jackets. Part A: Experimental study." Construct. Build. Mater., 19(3), 223-232.

Bisby, L. A., Dent, A. J. S., and Green, M. F. (2005). "Comparison of confinement models for fiber-reinforced polymer-wrapped concrete." ACI Struct. J., 102(1), 62-72.

Carey, S. A., and Harries, K. A. (2005). "Axial behavior and modeling of confined small-, medium-, and large-scale circular sections with carbon fiber-reinforced polymer jackets." ACI Struct. J., 102(4), 596-604.

Chaallal, O., Shahawy, M., and Hassan, M. (2003). "Performance of axially loaded short rectangular columns strengthened with carbon fiberreinforced polymer wrapping." J. Compos. Construct., 10.1061/ (ASCE) 1090-0268(2003)7:3(200), 200-208.

Csuka, B., and Kollár, L. P. (2012). "Analysis of FRP confined columns under eccentric loading." Compos. Struct., 94(3), 1106-1116.

Cusson, D., and Paultre, P. (1995). "Stress-strain model for confined highstrength concrete." J. Struct. Eng., 10.1061/(ASCE)0733-9445(1995) 121:3(468), 468-477.

De Lorenzis, L., Micelli, F., and La Tegola, A. (2002). "Influence of specimen size and resin type on the behavior of FRP-confined concrete cylinders." Proc., 1st Int. Conf., Advanced Polymer Composites for Structural Applications in Construction, R. A. Shenoi, S. S. J. Moy, and L. C. Hollaway, eds., Thomas Telford Publishing, London, 231-239.

De Lorenzis, L., and Tepfers, R. (2003). "Comparative study of models on confinement of concrete cylinders with fiber-reinforced polymer composites." J. Compos. Construct., 7(3), 10.1061/(ASCE)10900268(2003)7:3(219), 219-237.

Demers, M., and Neale, K. W. (1994). "Strengthening of concrete columns with unidirectional composite sheets." Developments in short and medium span bridge engineering, A. A. Mufti, B. Bakht, and L. G. Jaeger, eds., Canadian Society for Civil Engineering, Montreal, 895-905.

Hadi, M. N. S., Pham, T. M., and Lei, X. (2013). "New method of strengthening reinforced concrete square columns by circularizing and wrapping with fiber-reinforced polymer or steel straps." J. Compos. Construct., 10.1061/(ASCE)CC.1943-5614.0000335, 229-238. 
Ilki, A., and Kumbasar, N. (2003). "Compressive behaviour of carbon fibre composite jacketed concrete with circular and non-circular crosssections." J. Earthquake Eng., 7(3), 381-406.

Ilki, A., Peker, O., Karamuk, E., Demir, C., and Kumbasar, N. (2008). "FRP retrofit of low and medium strength circular and rectangular reinforced concrete columns." J. Mater. Civ. Eng., 10.1061/(ASCE)0899-1561(2008)20:2(169), 169-188.

Jiang, T., and Teng, J. G. (2007). "Analysis-oriented stress-strain models for FRP-confined concrete.” Eng. Struct., 29(11), 2968-2986.

Karabinis, A., Rousakis, T., and Manolitsi, G. (2008). "3D finite-element analysis of substandard RC columns strengthened by fiber-reinforced polymer sheets." J. Compos. Construct., 10.1061/(ASCE)1090-0268 (2008)12:5(531), 531-540.

Karabinis, A. I., and Rousakis, T. C. (2002). "Concrete confined by FRP material: A plasticity approach.” Eng. Struct., 24(7), 923-932.

Karbhari, V. M., and Gao, Y. (1997). "Composite jacketed concrete under uniaxial compression verification of simple design equations." J. Mater. Civ. Eng., 10.1061/(ASCE)0899-1561(1997)9:4(185), 185-193.

Lam, L., and Teng, J. G. (2003a). "Design-oriented stress-strain model for FRP-confined concrete." Constr. Build. Mater., 17(6-7), 471-489.

Lam, L., and Teng, J. G. (2003b). "Design-oriented stress-strain model for FRP-confined concrete in rectangular columns." J. Reinforc. Plast. Compos., 22(13), 1149-1186.

Lam, L., Teng, J. G., Cheung, C. H., and Xiao, Y. (2006). "FRP-confined concrete under axial cyclic compression." Cement Concr. Compos., 28(10), 949-958.

Lu, G., and Yu, T. X. (2003). Energy absorption of structures and materials, Woodhead Publishing, Boca Raton, FL.

Mander, J. B., Park, R., and Priestley, M. J. N. (1988). "Theoretical stressstrain model for confined concrete.” J. Struct. Eng., 10.1061/(ASCE) 0733-9445(1988)114:8(1804), 1804-1826.

Masia, M. J., Gale, T. N., and Shrive, N. G. (2004). "Size effects in axially loaded square-section concrete prisms strengthened using carbon fibre reinforced polymer wrapping." Can. J. Civ. Eng., 31(1), 1-13.

Matthys, S., Taerwe, L., and Audenaert, K. (1999). "Tests on axially loaded concrete columns confined by fiber reinforced polymer sheet wrapping." Proc., 4th Int. Symp. on Fiber Reinforced Polymer Reinforcement for Reinforced Concrete Structures, C. W. Dolan, S. H. Rizkalla, and S. H. Nanni, eds., 188, American Concrete Institute, Farmington Hills, MI, 217-229.

Mirmiran, A., Shahawy, M., Samaan, M., Echary, H. E., Mastrapa, J. C., and Pico, O. (1998). "Effect of column parameters on FRP-confined concrete." J. Compos. Construct., 10.1061/(ASCE)1090-0268(1998) 2:4(175), 175-185.

Miyauchi, K., Inoue, I., Kuroda, T., and Kobayashi, A. (1999). "Strengthening effects of concrete column with carbon fiber sheet." Trans. Japan Concr. Inst., 21(1), 143-150.

Parvin, A., and Wang, W. (2001). "Behavior of FRP jacketed concrete columns under eccentric loading." J. Compos. Construct., 10.1061/ (ASCE)1090-0268(2001)5:3(146), 146-152.

Pessiki, S., Harries, K. A., Kestner, J. T., Sause, R., and Ricles, J. M. (2001). "Axial behavior of reinforced concrete columns confined with FRP jackets." J. Compos. Construct., 10.1061/(ASCE)1090-0268 (2001)5:4(237), 237-245.

Richart, F. E., Brandtzaeg, A., and Brown, R. L. (1929). "The failure of plain and spirally reinforced concrete in compression." Bulletin 1990, Univ. of Illinois Engineering Experimental Station, Champaign, IL.

Rochette, P., and Labossiére, P. (2000). "Axial testing of rectangular column models confined with composites." J. Compos. Construct., 10.1061/(ASCE)1090-0268(2000)4:3(129), 129-136.

Rousakis, T. C., and Karabinis, A. I. (2012). "Adequately FRP confined reinforced concrete columns under axial compressive monotonic or cyclic loading." Mater. Struct., 45(7), 957-975.
Rousakis, T. C., Karabinis, A. I., and Kiousis, P. D. (2007). "FRP-confined concrete members: Axial compression experiments and plasticity modelling." Eng. Struct., 29(7), 1343-1353.

Rousakis, T. C., Rakitzis, T., and Karabinis, A. I. (2012). "Empirical modelling of failure strains of uniformly FRP confined concrete columns." Proc., Int. Conf. on FRP Composites in Civil Engineering, Int. Institute for FRP in Construction, Queen's Univ., Kingston, ON, 1-8.

Saadatmanesh, H., Ehsani, M. R., and Li, M. W. (1994). "Strength and ductility of concrete columns externally reinforced with fiber-composite straps." ACI Struct. J. 91(4), 434-447.

Saenz, N., and Pantelides, C. (2006). "Short and medium term durability evaluation of FRP-confined circular concrete." J. Compos. Construct., 10.1061/(ASCE)1090-0268(2006)10:3(244), 244-253.

Shehata, I. A. E. M., Carneiro, L. A. V., and Shehata, L. C. D. (2002). "Strength of short concrete columns confined with CFRP sheets." Mater. Struct., 35(1), 50-58.

Suter, R., and Pinzelli, R. (2001). "Confinement of concrete columns with FRP sheets." Proc., Int. Symp. on Fiber-Reinforced Polymer Reinforcement for Concrete Structures, C. Burgoyne, ed., Thomas Telford Publishing, London, 793-802.

Tao, Z., Yu, Q., and Zhong, Y. Z. (2008). "Compressive behaviour of CFRP-confined rectangular concrete columns." Mag. Concr. Res., 60(10), 735-745.

Tasdemir, M. A., Tasdemir, C., Akyüz, S., Jefferson, A. D., Lydon, F. D., and Barr, B. I. G. (1998). "Evaluation of strains at peak stresses in concrete: A three-phase composite model approach." Cement Concr. Compos., 20(4), 301-318.

Teng, J. G., Jiang, T., Lam, L., and Luo, Y. Z. (2009). "Refinement of a design-oriented stress-strain model for FRP-confined concrete." J. Compos. Construct., 10.1061/(ASCE)CC.1943-5614.0000012, 269-278.

Toutanji, H. A. (1999). "Stress-strain characteristics of concrete columns externally confined with advanced fiber composite sheets." ACI Mater. J., 96(3), 397-404.

Valdmanis, V., De Lorenzis, L., Rousakis, T., and Tepfers, R. (2007). "Behaviour and capacity of CFRP-confined concrete cylinders subjected to monotonic and cyclic axial compressive load." Struct. Concr., $8(4), 187-190$

Wang, L. M., and Wu, Y. F. (2008). "Effect of corner radius on the performance of CFRP-confined square concrete columns: Test." Eng. Struct., 30(2), 493-505.

Watanabe, K., et al. (1997). "Confinement effect of FRP sheet on strength and ductility of concrete cylinders under uniaxial compression." Proc., Int. Symp., Non-Metallic (FRP) Reinforcement for Concrete Structures, Vol. 1, Japan Concrete Institute, Sapporo, Japan, 233-240.

Wolff, C., Bastid, P., and Bunsell, A. R. (1994). "Relation of energy absorption of composite structures to material strength." Compos. Eng., 4(2), 195-218.

Wu, Y. F., and Wei, Y. Y. (2010). "Effect of cross-sectional aspect ratio on the strength of CFRP-confined rectangular concrete columns." Eng. Struct., 32(1), 32-45.

Wu, Y. F., and Zhou, Y. W. (2010). "Unified strength model based on HoekBrown failure criterion for circular and square concrete columns confined by FRP." J. Compos. Construct., 10.1061/(ASCE)CC.19435614.0000062, 175-184.

Xiao, Y., and Wu, H. (2000). "Compressive behavior of concrete confined by carbon fiber composite jackets." J. Mater. Civ. Eng., 10.1061/ (ASCE)0899-1561(2000)12:2(139), 139-146.

Yazici, V., and Hadi, M. N. S. (2012). "Normalized confinement stiffness approach for modeling FRP-confined concrete." J. Compos. Construct., 10.1061/(ASCE)CC.1943-5614.0000283, 520-528. 